\title{
Plastic zone size at notch tip in mode I fracture using the modified Irwin's model and volumetric approach
}

\author{
Mouna FAKIR ${ }^{\mathrm{a}}$,Hassan EL MINOR ${ }^{\mathrm{a}}$, Hanane EL MINOR \\ ${ }^{a}$ Research Team Materials, Mechanics and Civil Engineering, ENSAAGADIR, 80000,Morocco \\ ${ }^{b}$ Polytechnique Agadir, MEER, Universiapolis Bab Al Madina, Agadir 80000, Maroc.
}

\begin{abstract}
At crack tip the stress distribution has singularities (i.e the stress intensity is infinite) which is physically impossible. Irwin realised that the stresses at crack tip are finite and subsequently introduced the Stress Intensify Factor(SIF). This factor is used to determine this plastic zone size. The crack is a special case of the notch. Around the notch, the fracture needs a volume to develop according to the volumetric approach. This volume is characterised by the distance effective Xeff, the stress effective $\sigma_{\text {eff }}$ and the stress intensity factor $K_{\rho}^{I}$. In This paper a new proposed formula called “Modified Irwin's Model” based on Irwin's model can be used to determine the plastic zone size. This new formula will be verified using numerical finite element simulation and validated using the Von-Mises and Tresca Criteria. The Modified Irwin's Model can be an alternative way to estimate the fracture elaboration zone from the plastic zone.
\end{abstract}

Keywords: Notch; Volumetric Approach; Irwin's model; Effective distance; Plastic zone; Mode I fracture

\section{Introduction}

Any structure contains defects, whether they are introduced during the elaboration of the material or during the manufacturing (machining or welding defects for example). These defects assumed to cracks, must be taken into account because they determine the reliability of the structure. It is the objective of fracture mechanics which was introduced by GRIFITH [1] in 1920. He quantified a global variable related to the fracture which will be called later the energy restitution rate. In 1957, IRWIN [2] introduced the notion of stress intensity factor (SIF) to explain the singularity phenomenon around the crack tip. This means that the stresses at the crack tip are finite which creates a plastic zone. To estimate this zone, two criteria are used: Von Mises criterion and Tresca criterion. However, considering any defect as a crack is conservative, and also experimenting on pre-cracked specimens is very expensive where from the appearance of the notion of "Notch". The notch is characterised by the following geometric parameters: the length " $a$ ", the angle " $\psi$ " and the radius " $\rho$ " [3]. The notch effect $[4,5]$ determines the behaviour of the local notch area. The notch geometry, the boundary conditions and the local material response identify the notch effect [6]. Several approaches have been studied to delimit the shape of this zone. In this paper, the volumetric approach is used. This approach assumes that the fracture elaboration needs a physical volume which named "effective volume" to engender. This volume is characterized by the effective distance and the effective stress [7].

Several research $[23,24,25,26]$ are focused on the determination of the plastic zone but the notion of the fracture process zone is rarely developed [17]. In this paper, the plastic zone found by the Irwin's model and the fracture elaboration zone assumed by the volumetric approach will be studied and compared using the formula proposed at the base of the Irwin's model. This comparison will make it possible to confront these two physical notions. This proposed formula based on the Irwin model which allows to determine the plastic zone, it will be verified using numerical simulation by the finite element method. Also a relation between this modified Irwin model and the effective distance will be proposed and verified. This will allow to find an alternative way to determine the plastic zone.

\section{Volumetric approach}

The volumetric approach is a local criterion which assumes that the fracture is elaborated in a physical volume [8]. In this volume, the effective stress is assumed to be equal to an average stress [7]. According to [8,9] this volume is considered cylindrical and its diameter from the notch tip is called the effective distance Xeff. The relative stress gradient $\chi(r)$ is used to define this effective distance Xeff according to the equation below [10, 11]: 


$$
\chi(r)=\frac{1}{\sigma_{y y}(r)} \frac{\partial \sigma_{y y}(r)}{\partial r}(1)
$$

Which $\sigma_{\mathrm{yy}}(\mathrm{r})$ is the normal stress and $\chi(\mathrm{r})$ is the relative stress gradient and

The effective distance Xeff is the distance which the relative stress gradient reaches its minimum [12, 13]. In several investigation $[13,14,15]$ the distribution of elastic-plastic stress is studies as illustrated in Fig.1.

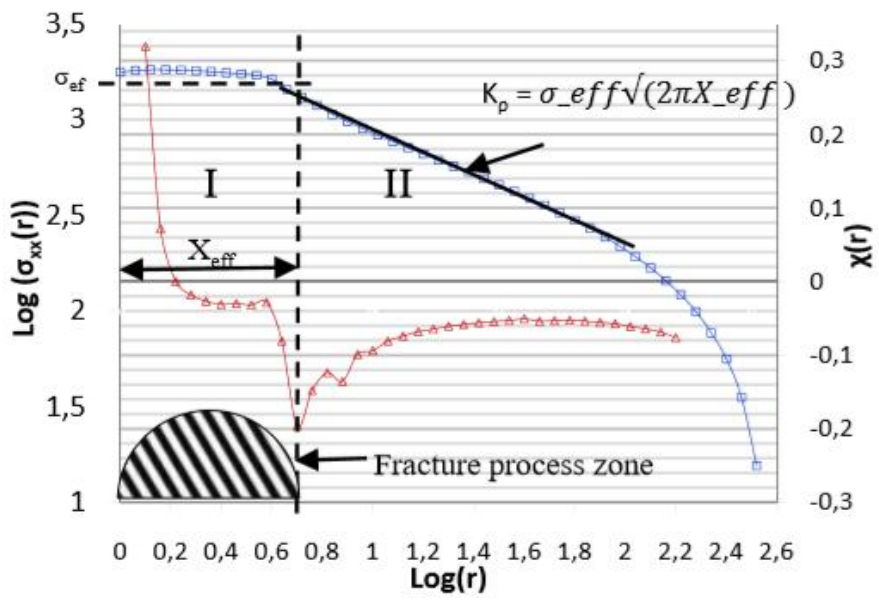

Fig. 1Elastic-plastic stress filed and the effective distance Xefffrom notch tip

As shown in Fig.1, the graph is divided on two zones:

- Zone I: The elastic-plastic stress distribution increases and reach the pick value then drops gradually in the elastic filed.

- Zone II: this zonestarts from an effective distance and the curve shows the linear behaviour of the stress filed in the bi logarithmic diagram

The effective distance represents the limit of the fracture process elaboration zone. From which the stress intensity factor (SIF) is specified by this distance [7,9]. As mentioned in the section above, the stress effective is equal to the average of the stress distribution in this volume. Also to take account of geometry of notch and the loading mode, the average stress is multiplied by the weight function.

According to [5] the formula's effective stress is :

$$
\sigma_{\text {eff }}=\frac{1}{\mathrm{X}_{\mathrm{eff}}} \int_{0}^{\mathrm{Xeff}} \sigma_{\mathrm{yy}}(\mathrm{r}) \varphi(\mathrm{r}) \mathrm{dr}
$$

With $\varphi(r)$ is the weight function wich have for expression [16]:

$$
\varphi(r)=1-r \chi(r)
$$

With $\chi(r)$ is the relative stress gradient.

The stress intensity factor is calculated only in the area where is characterized by effective distance [7,9]:

$$
K_{\rho}^{I}=\sigma_{e f f} \sqrt{2 \pi X_{e f f}}(4)
$$

With $\sigma_{e f f}$ is effective stress and $X_{\text {eff }}$ is effective distance.

The volumetric approach is the most appropriate approach to specify the extent of the fracture elaboration volume. However, the size of the plastic zone must also be known. The relation between the two zones (the fracture elaboration zone and the plastic zone) allows an understanding of the local behaviour around the notch tip. 


\section{Material and methodology}

\subsection{Material}

Aluminum alloy has a wide range of uses and applications in our daily lives. It is the material of the future. As result, the in-depth study of its behavior has become necessary to optimize its dimensioning and subsequently its cost. In this paper, the material used for modeling is the aluminum alloy Al 5083 according to [19, 20]. The Fig. 2 shows the stress strain curves of the Al 5083 (engineering and the true curve). As the Fig.2 shown, Al 5083 have a plastic behavior.

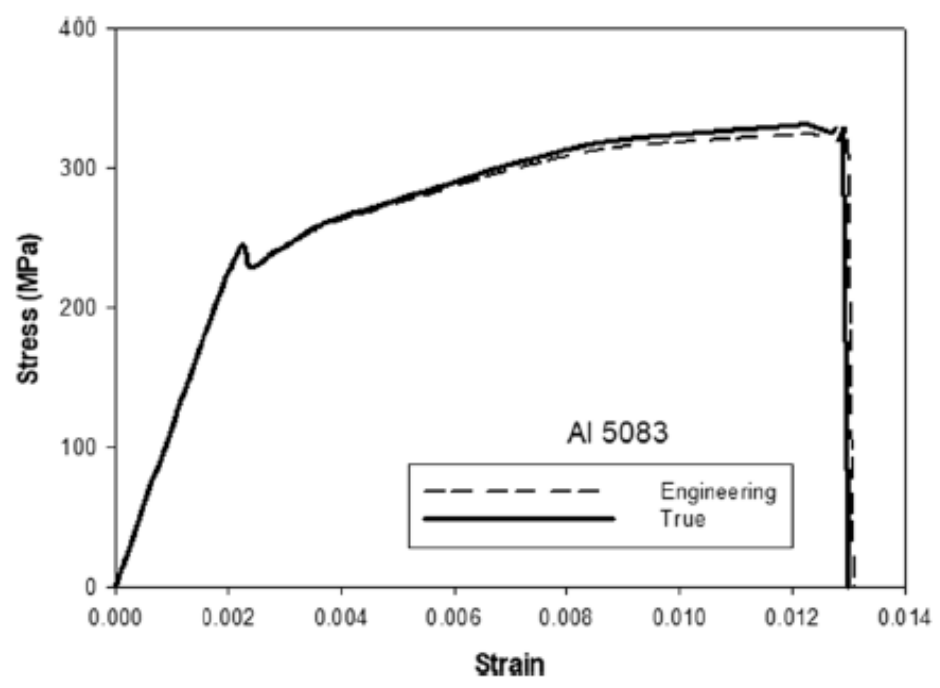

Fig. 2Stress-strain curves (engineering and the true curve) for the Al $5083[19,20]$

The mechanical proprieties are summarized in the Table $1[19,20]$ :

Table1:Mechanical proprieties of the AL 50830 [19,20]
\begin{tabular}{llll}
\hline $\mathrm{E}(\mathrm{GPa})$ & $\mathrm{Ru}(\mathrm{Mpa})$ & $\mathrm{Re}(\mathrm{Mpa})$ & $\mathrm{v}$ \\
\hline 67 & 330 & 225 & 0.2 \\
\hline
\end{tabular}

With $\mathrm{E}$ is the Elastic modulus, $\mathrm{Ru}$ is the ultimate tensile strength, Re is the tensile yield strength and $v$ is the Poisson's ratio.

\subsection{Specimen}

In this investigation, four rectangular specimens are used [19]. The specimens are central U-notched. The geometry conditions are as follow:

The width is $60 \mathrm{~mm}$ the length is $200 \mathrm{~mm}$, the thickness is $5 \mathrm{~mm}$ and the notch length is $30 \mathrm{~mm}$ [19]. The notch radius has the following values: $\rho=4 \mathrm{~mm}, 2 \mathrm{~mm}, 1 \mathrm{~mm}$ and $0.5 \mathrm{~mm}$ [19]. As illustrated in the Fig3, the width and the length are far bigger than the thickness of the specimen. Consequently the specimens are studied under plane-stress conditions. Also, given the loading conditions, these specimens are considered under mode I fracture. 
Fig. 3Tensile rectangular

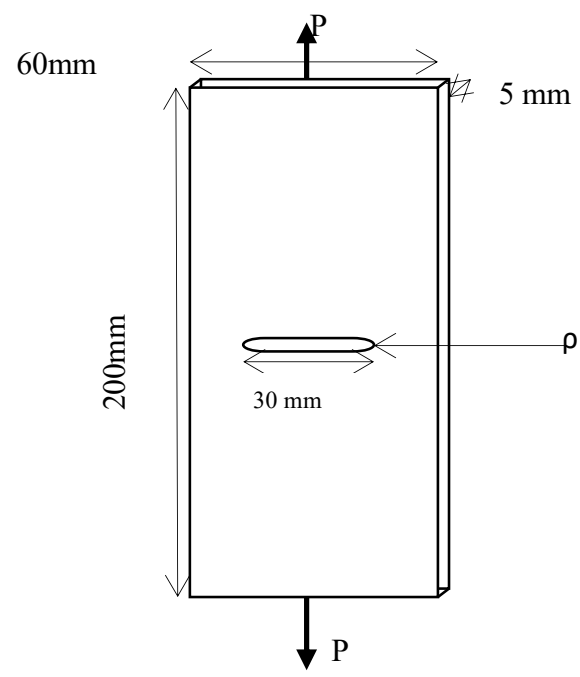

specimen U-notched

The specimens are analysed in two steps: the first one is to apply various external load values in order to know the impact of the external load on the stress fields, and the second step is to consider the following loads $20 \mathrm{KN}, 22 \mathrm{KN}$, $24 \mathrm{KN}$, and $23.5 \mathrm{KN}$ for the notch tip radii $0.5,1,2$ and $4 \mathrm{~mm}$ respectively [19].

\subsection{Numerical simulation - finite elements}

The quarter of the specimen has been modelled as shown in Fig. 4 because the specimen is rectangular and symmetric. To reduce the calculation time, the quarter of the specimen is in 2D. To model the quarter of the specimen, the following boundary condition must be applied:

- $\quad \mathrm{Ux}=\mathrm{Uy}=\mathrm{Ry}=0$ on line $\mathrm{BC}$ (Fig.4) with Ux and $\mathrm{Uy}$ are the displacement along the $\mathrm{X}$ and $\mathrm{Y}$ axis respectively, and $\mathrm{Ry}$ is the rotation related to $\mathrm{Y}$ axis.

- $\mathrm{Uy}=\mathrm{Uz}=\mathrm{Rx}=0$ on line $\mathrm{ED}$ (Fig.4) with $\mathrm{Uy}$ and $\mathrm{Uz}$ are the displacement along the $\mathrm{Y}$ and $\mathrm{Z}$ axis respectively, and $\mathrm{Rx}$ is the rotation related to $\mathrm{X}$ axis.

The property of $\mathrm{Al} 50883$ introduced in the software respected the plastic behaviour presented in Fig.2. As the analysis is under plane-stress conditions, the thickness is considered equal to $1 \mathrm{~mm}$. In Fig. 5, as illustrated, the mesh used is made up of the four nodes plane stress element, i.e CPS4R. The software used in this paper was used in several research $[21,22]$. To optimise the mesh, around the notch the approximate element size is 0.05 , and it equal to 1 away from the notch, this way the calculations do not take much time. The software used allows determining the plastic zone size and the elaboration of fracture zone [23]. Several researchers [19-23] have used this software for the same purpose and the results found numerically and those found analytically are very close.

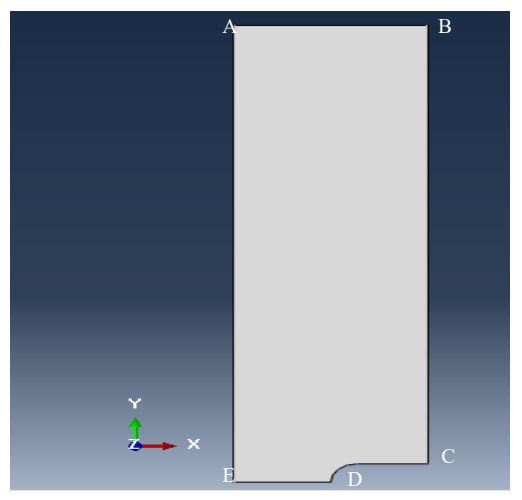

Fig. 4 The quarter of the rectangular specimen modelled.

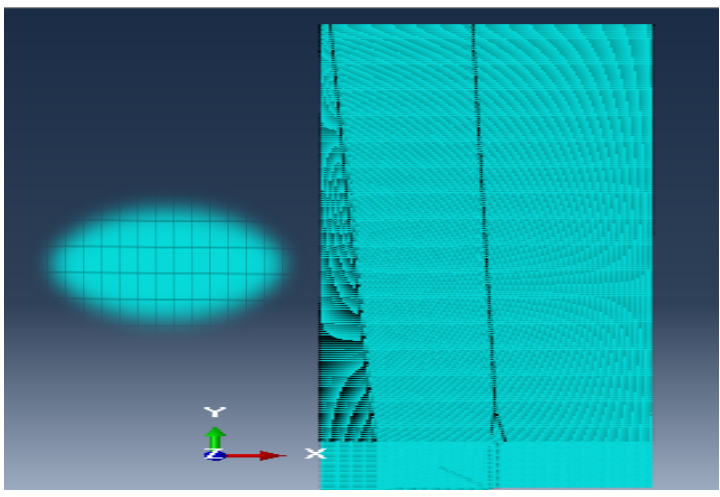

Fig. 5 Mesh used in modelling

Following a singularity of the stress filed around the notch tip, the mesh is very refined in this region as shown in the 
Fig. 6 to increase the accuracy of results. In Fig. 7 the plastic zone is presented around the notch tip obtained by FE.
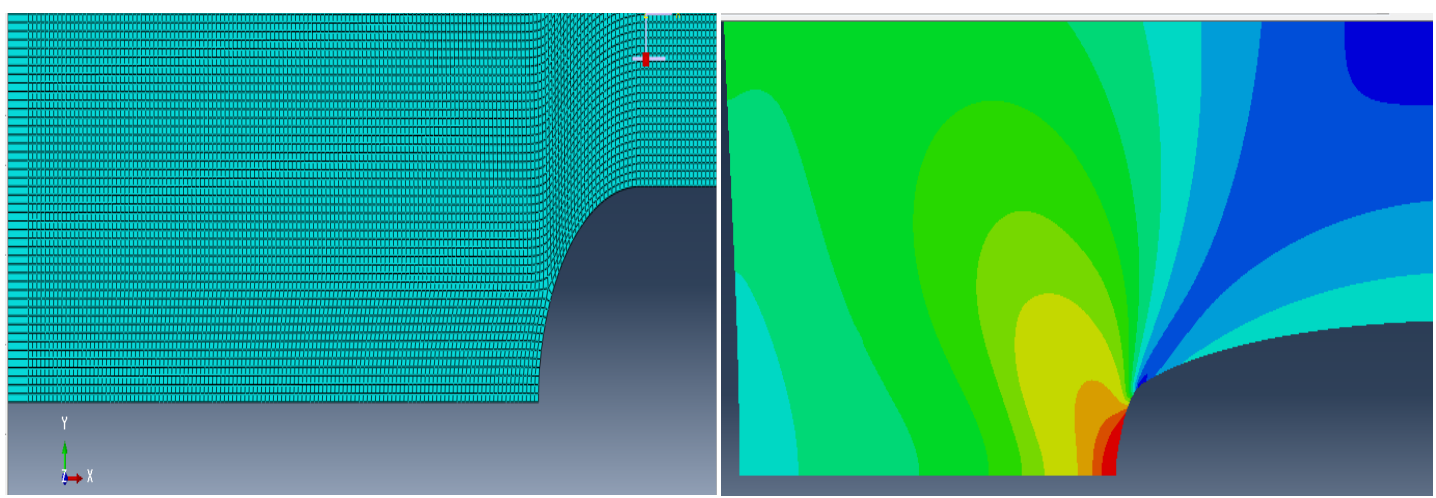

Fig. 6 Refining mesh around the notch tip.Fig. 7Plastic zone around the notch tip.

\section{Influence of the external load on the stress fields}

For the four specimens (i.e. the four notch radii), different external loads are applied, namely: $20 \mathrm{KN}, 22 \mathrm{KN}, 23.5$ $\mathrm{KN}, 24 \mathrm{KN}, 25 \mathrm{KN}, 26 \mathrm{KN}, 27 \mathrm{KN}$ and $28 \mathrm{KN}$.

As presented in Fig. 8 the stress distribution fields increase with the load. This means that as the external load increases, the local stress (i.e. $\sigma_{y y}$ in this case) also increases regardless of the notch radius. In another research [27], E. Smith found that in mode I the local stress $\left(\sigma_{\mathrm{yy}}\right)$ near a circular cylindrical notch depends on the loading characteristics.

According to the Fig.8, the stress intensity increases if the distance $r$ from the notch tip and the notch radii decrease ( $\mathrm{r}$ : is the distance from the notch tip (D) in the ligament (ED) in Fig. 3). This explains the stress concentration around the notch.
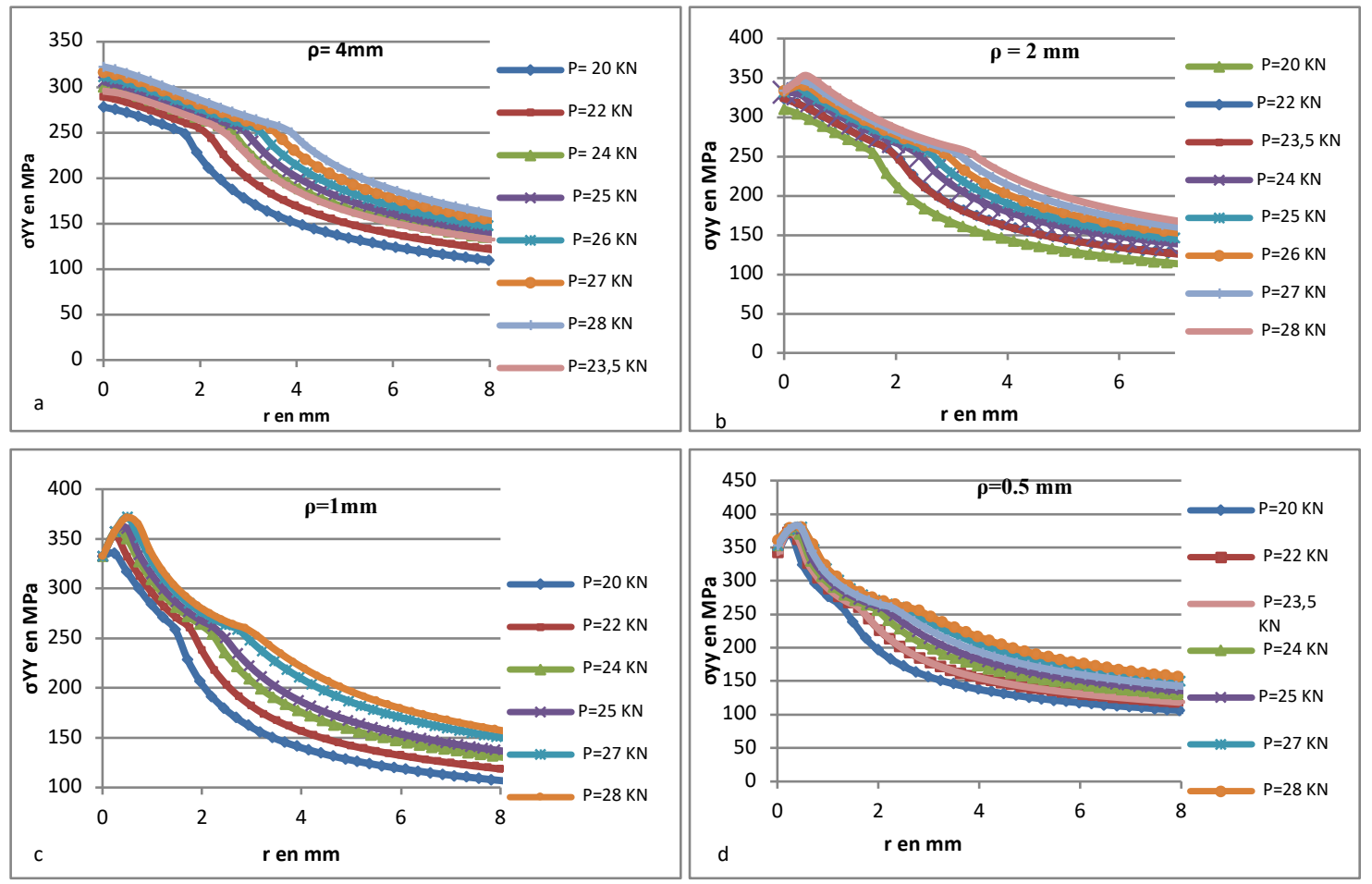

Fig. 8Stress distribution fields versus $r$ for $a: \rho=4 \mathrm{~mm}, b: \rho=2 \mathrm{~mm}, c: \rho=1 \mathrm{~mm}$ and $d: \rho=0.5 \mathrm{~mm}$ 


\section{Plastic zone and modified Irwin's model}

\subsection{Irwin's model}

As studied by Irwin [2], the presence of singularities in the stress distribution field at the crack tip makes this behaviour not physically acceptable. Irwin introduced the SIF to determine the plastic zone at the crack tip according to the following formula [18]:

$$
R_{P}=\frac{1}{2 \pi}\left(\frac{K_{I}}{R_{e}}\right)^{2}
$$

With $\mathrm{Rp}$ is the diameter of the plastic zone, $\mathrm{K}_{\mathrm{I}}$ is the stress intensity factor (SIF) in mode I fracture and $R_{e}$ is the yield strength.

From the above equation Irwin has assumed the plastic zone to be circular.

\subsection{Modified Irwin's model}

This paper proposes a new formula based on the Irwin model (presented in the previous section) to determine the plastic zone. This new formula applied on the notch tip generalizes the particular case of the crack. The modified Irwin's model is presented as follows:

$$
R_{P I M}=\frac{1}{2 \pi}\left(\frac{K_{\rho}^{I}}{\sigma_{e f f}}\right)^{2}
$$

With $K_{I}^{\rho}$ is the SIF in mode I and $\rho$ is the radius of the notch $\sigma_{e f f}$ is the effective stress. Analytically and according to the equation (4), the equation (6) becomes:

$$
R_{P I M}=\frac{1}{2 \pi}\left(\frac{\sigma_{e f f} \sqrt{2 \pi X_{e f f}}}{\sigma_{e f f}}\right)^{2}
$$

With all due simplifications equation (7) becomes:

$$
R_{P I M}=X_{\text {eff }}
$$

Using the modified Irwin's model and according to the equation (8) it is shown that the plastic zone is equal to the fracture elaboration zone

In order to validate this proposed formula of the modified Irwin model, numerical simulations using appropriate software on notched specimens were performed. The following section presents the methodology of these simulations.

\subsection{Von Mises and Tresca criterion}

According to the Von Mises and Tresca criteria, the plastic zone is determined if the stress reaches yield stress of the material.

The formula of Von Mises criterion is :

$$
\sigma_{\mathrm{VM}}=\frac{1}{\sqrt{2}} \sqrt{\left(\sigma_{\mathrm{xx}}-\sigma_{\mathrm{yy}}\right)^{2}+\left(\sigma_{\mathrm{xx}}-\sigma_{\mathrm{zz}}\right)^{2}+\left(\sigma_{\mathrm{zz}}-\sigma_{\mathrm{yy}}\right)^{2}}
$$

With $\sigma_{\mathrm{xx}}, \sigma_{\mathrm{yy}}, \sigma_{\mathrm{zz}}$ are the tree principal stresses. Under the plane stress condition $\sigma_{\mathrm{zz}}=0$ then the equation (9) becomes:

$$
\sigma_{\mathrm{VM}}=\frac{1}{\sqrt{2}} \sqrt{\left(\sigma_{\mathrm{xx}}-\sigma_{\mathrm{yy}}\right)^{2}+\sigma_{\mathrm{xx}}^{2}+{\sigma_{\mathrm{yy}}}^{2}}
$$

The formula of Tresca criterion is:

$$
\operatorname{Max}\left(\left|\sigma_{x x}-\sigma_{y y}\right|,\left|\sigma_{y y}-\sigma_{z z}\right|,\left|\sigma_{z z}-\sigma_{x x}\right|\right) \geq \sigma_{y}
$$

With $\sigma_{\mathrm{xx}}, \sigma_{\mathrm{yy}}, \sigma_{\mathrm{zz}}$ are the tree principal stresses and $\sigma_{y}$ yield stress of the material. Under the plane stress conditions $\sigma_{\mathrm{zz}}=0$ then the equation (9) becomes: 


$$
\operatorname{Max}\left(\left|\sigma_{x x}-\sigma_{y y}\right|,\left|\sigma_{y y}\right|,\left|\sigma_{x x}\right|\right) \geq \sigma_{y}
$$

And since the $\sigma_{y y}$ is the predominant stress the formula (9) becomes :

$$
\left|\sigma_{y y}-0\right| \geq \sigma_{y}
$$

\section{Results and discussion}

In order to study the modified Irwin's model (6) and to get a small or moderate plastic zone size around the notch tip [19], the specimen is submitted to external tensile load (P) : $20 \mathrm{KN}, 22 \mathrm{KN}, 24 \mathrm{KN}$ and $23.5 \mathrm{KN}$ for the notch tip radii $0.5 \mathrm{~mm}, 1 \mathrm{~mm}, 2 \mathrm{~mm}$ and $4 \mathrm{~mm}$ respectively [19]. The modified Irwin's model is compared to Von Mises and Tresca criterion. The discrepancy $(\Delta)$, which is presented in equation (14), is used to understand the differences between the different results:

$$
\Delta=\frac{(P Z S)_{F E}-(P Z S)_{I M}}{(P Z S)_{F E}}(14)
$$

It is interesting to mention that only the PZS on the notch ligament (i.e. the effective PZS) is used for comparing the results. Because under the mode I of fracture the effective PZS is used to determine the fracture behaviour in notched behaviour [19].

\subsection{Results of the Fracture Elaboration Zone using the volumetric approach}

The estimation of the fracture elaboration zone is based on the volumetric approach [8]. The effective distance Xeff using the relative stress gradient is presented in Fig 9 for different values of $\rho$.
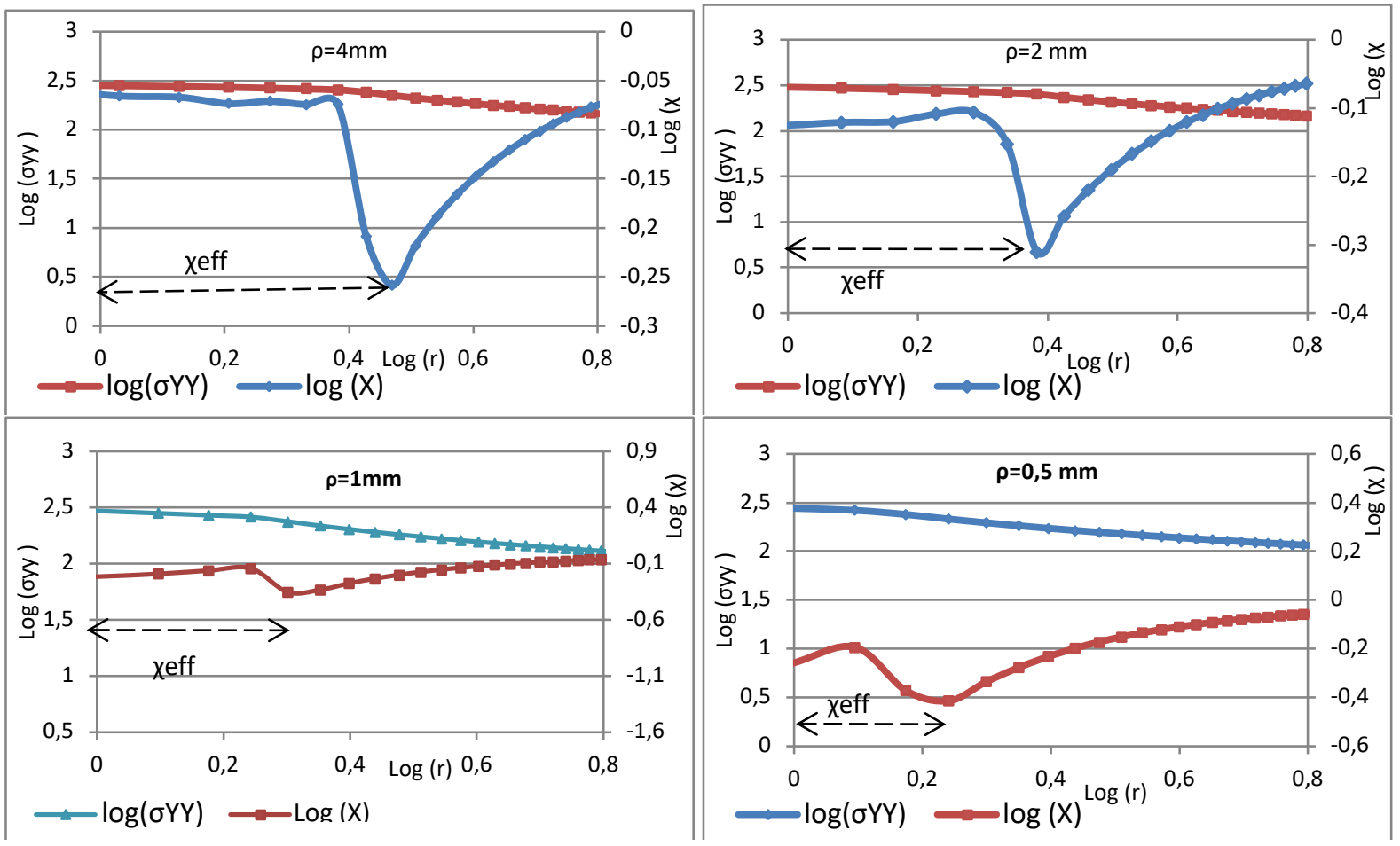

Fig. 9 Effective distances for various values of $\rho$

The effective distance, the effective stress $\sigma_{\text {eff }}$ and the stress intensity factor $K_{\rho}^{I}$ values [7,9], according to the equations (1), (2) and (3) are summarised in the Table 2: 
Table 2 Values of the effective distanceXeff, the effective stress $\sigma_{\text {eff }}$ and the stress intensity factor $K_{\rho}^{I}$

\begin{tabular}{cccc}
\hline$\rho$ & Xeff & $\sigma$ eff & $\boldsymbol{K}_{\boldsymbol{\rho}}^{\boldsymbol{I}}$ \\
\hline 0,5 & 1,49 & 271,73 & 26,30 \\
1 & 1,74 & 285,19 & 29,89 \\
2 & 2,49 & 261,36 & 32,74 \\
4 & 2,67 & 253,84 & 32,91 \\
\hline
\end{tabular}

\subsection{Results of the plastic zone using the modified Irwin's model}

As shown in Fig.10 the plastic zone estimated by the proposed modified Irwin's model is very close to the one calculated by Von Mises and TrescaCriterion.

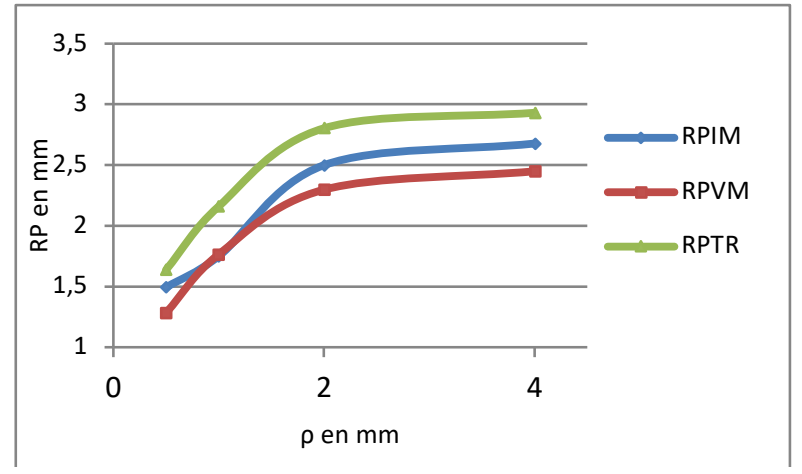

Fig. 10 Comparison of the plastic zone estimated by Modified Irwin's Model and the Von Mises and Tresca Criterion

With RPIM is the size of the plastic zone using the modified Irwin's model, RPVM is the plastic zone size using the Von Mises criterion and RPTR is the size of the plastic zone using the Tresca Criterion.

To verify this new formula of modifies Irwin's model, the Table 2 shows the discrepancy $(\Delta)$ for the tree results:

Table 3: Values of the effective PZS based on Von Mises and Tresca criteria and the discrepancies

\begin{tabular}{cccc|cccc|cccc}
\hline \multicolumn{4}{c|}{ Von-Mises ctriterion } & \multicolumn{4}{c|}{ Tresca criterion } & \multicolumn{3}{c}{ Von Mises criterion compared to } \\
Tresca criterion
\end{tabular}

From the Table 3, in the first place it is noticed that the extent of the plastic zone increases with the radius of the notch. This means that the plastic zone depends on the notch's radius. Secondly to understand the results found of the discrepancy $(\Delta)$, a comparison between the standard plasticity criteria Von Mises and Tresca is added in the table 3. As the criteria Von Mises and Tresca are validated in plasticity behaviour, the discrepancy $(\Delta)$ between these two criteria reaches $21.62 \%$ as maximum values. Than the discrepancy less than $21.62 \%$ is considered acceptable and the results are verified. According to [19], the results are considered excellent if the discrepancy is less than 15\%. Finally, it is noted that the discrepancy between the PZS by modified Irwin's model and Von Mises Criterion is less than 15\% 
and that Tresca criterion is approximately 15\%, this leads to the conclusion that the modified Irwin's model is verified. And the results are encouraging to use the proposed formula.

\section{Conclusion}

The investigation presented in this paper concludes that:

- The variation in the magnitude of the applied external load has a direct impact on the stress distribution field around the notch. Also the stress intensity around the notch increases if the radius's notch is infinitely small, which implies that the crack (which is a special case of the notch when $\rho=0$ ) is the most dangerous defect and can induce fracture rapidly.

- Based on the volumetric approach, the fracture elaboration zone is characterised by Xeff (effective distance), $\sigma \operatorname{eff}$ (effective stress) and $K_{\rho}^{I}$ (stress intensity factor). These parameters depend on the geometry of the notch, the applied external loads and the boundary condition. They aren't the intrinsic parameters of the material.

- The modified Irwin's model proposed by the equation: $R_{P I M}=\frac{1}{2 \pi}\left(\frac{K_{I}^{\rho}}{\sigma_{e f f}}\right)^{2}$ is verified compared to standard plasticity models. Since as the discrepancy $(\Delta)$ between the results of plastic zone size given by the modified Irwin's model and Von Mises or Tresca criterion is approximately 15\% [19].

- The proposed modified Irwin's model can be used to determine the fracture elaboration zone according to the following relationship: $R_{P I M}=X_{e f f}$.

\section{References}

1. Alan Arnold Grifith.(The Phenomena of Rupture and Flow in Solids) Philosophical Transactions of the Royal Society A.221 pp163-198, (1920).

2. G.R. Irwin (Analysis of stresses and strains near the end of a crack traversing a plate) Journal of Applied Mechanics, Volume:2 (1957).

3. G.Pluvinage and M. Gjonaj ( Notch effect in fatigue and fracture),1-22, Kluwer Academic Publisher (2001)

4. I. Dlouhy, Z. Chlup, M. Holzmann, Local characteristics of (brittle) failure assessed from charpy type specimen, Notch Effects Fatigue Fract. (2001) 127- 146.

5. H. Adib-Ramezani, J. Jeong, Advanced volumetric method for fatigue life prediction sing stress gradient effects at notch roots, Comput. Mater. Sci. 39 (2007) 649-663.

6. L.Toth( the notch and size effect in brittle-ductile transition behaviour of materials)Bay Zoltán Institute of Logistics and Production SystemsMiskolc-TapolcaHungary (2001)

7. H. El Minor, A. Kifani, M. Louah, Z. Azari, G.Pluvinage ( Fracture toughness of high strength steel-using the notch stress intensity factor and volumetric approach) Elsevier, Structural safety 25 (2003)

8. A.CHACRONE (Plastic zone size and effective distance at notch root)R.F.M. Revue française de mécanique.( 2001), Num 1, pp 19-23

9. O.Zebri, H. El Minor, A. Benderma ( Plastic zone and effective distance under mixed mode fracture- volumetric approach-) journal of Mechanical and Civil Engineering-ISOR Journals, Vol.13 (2016)

10. G. Pluvinage, Z. Azari, N. Kadi, Effect of ferritic microstructure on local damage zone distance associated with fracture near notch, Theoret. ApplFract. Mech. 31(2) (1999) 149-156.

11. S.M. Khan, M.K. Khraisheh, Analysis of mixed mode crack initiation angles under various loading conditions, Eng. Fract. Mech. 67(5), 397-419, 2000.

12. G. Pluvinage, Fracture and Fatigue Emanating from stress concentrators" (Dordrecht: Kluwer Academic Publishers; 2003)

13. El-Hadim, (stress triaxiality to evaluate the effective distance in the volumetric approach in fracture mechanics) Journal of Mechanical and Civil Engineering, pp.21-26, (2011)

14. Al Gaoudi, O., Achemlal, I., El Minor, H., Pluvinage, G., (Plastic Zone and Volumetric Approach-Mixed Mode Fracture I+II Emanating From Notches, (2015) International Reviw of Mechanical Engineering (IREME), 9(2), pp. $160-166$ 
15. H. El Minor, An elliptical fracture criterion from mixed mode fractures I+II emanating from notches. Structural Engineering and Mechanics, Vol. 26, No. 1(2007)

16. Y. Weixing, Stress field intensity approach for predicting fatigue life, Int. J. Frac. 17 (4) (1995) 245-251.

17. M. FAKIR, H. El Minor, (The fracture process zone in mode I fracture emanating from notch and plastic zone size), Materials Today: Proceedings, (2020)

18. G.R. Irwin (Plastic zone near a crack and fracture toughness) Sagamore Res. Conf. Proc., 4 (1961), pp. 63-78

19. A.R. Torabi, M. Kamyab (Notch ductile failure with significant strain-hardening: The modified equivalent material concept )Fatigue Fract. Eng. Mater. Struct., 42 (2018), pp. 439-453

20. A.R. Torabi, B. Shahbazian ( Notch tip plastic zone determination by extending Irwin's model) theoretical and applied fracture mechanics, volume 108 (2020)

21. G.Z. Wang, X.L. Liu, F.Z. Xuan, S.T. Tu" Effect of constraint induced by crack depth on creep crack-tip stress field in CT specimens", International Journal of Solids and Structures, 47(2010)51-57

22. Deeprodyuti Sen, J. Chattopadhyay "New $\eta$-factor equation for evaluation of J-integral of shallow cracked CT specimen considering R-O material strain hardening", Theoretical and Applied Fracture Mechanics 97(2018) 98107

23. K.H. Benrahou, M. Benguediab, M. Belhouari, M. Nati-Abdelaziz, A. Imad, Estimation of the plastic zone by finite element method under mixed mode (I and II) loading, Sci. Direct Comput. Mater. Sci. 38(2007) 595-601

24. Tomas Vojtek, Miroslav Hrstka "How to get a correct estimate of the plastic zone size for shear-mode fatigue cracks?" Theoretical and Applied Fracture Mechanics, volume 104, December 2019.

25. Yaocai Ma, Aizhong Lu, Xiangtai Zeng, Hui Cai " Analytical solution for determining the plastic zones around twin circular tunnels excavated at great depth" International Journal of Rock Mechanics and Mining Sciences, Volume 136, December 2020.

26. Tito Andriollo, Varvara Kouznetsova "A simplified formula to estimate the size of the cyclic plastic zone in metals containing elastic particles" Engineering Fracture Mechanics, volume 241, January 2021.

27. E. Smith "The elastic stress distribution near a circular cylindrical notch due to external dislocations" International Journal of Engineering Science, volume 42, issues 17-18, octobre 2004, page 1841-1846 